\title{
Multiple Subcutaneous Nodules Leading to Diagnosis of Colon Cancer
}

\author{
Irappa Madabhavi ${ }^{1, *}$, Nagaveni Kadakol ${ }^{2}$, Chidanand Chavan ${ }^{3}$, Malay Sarkar ${ }^{3}$
}

1. Consultant Medical and Pediatric Oncologist and Hematologist, Kerudi Cancer Hospital, Bagalkot, Karnataka, India

2. Lecturer, Government Polytechnic College, Bilagi, Karanataka, India

3. Indira Gandhi Medical College, Shimla, Himachal Pradesh, India

* Corresponding Author:

Madabhavi Irappa Madabhavi, MD Kerudi Cancer Hospital, Bagalkot, Karnataka, India

Telefax: + 7619501323

Email: irappamadabhavi@gmail.com

Received: 28 Dec. 2017

Accepted: 22 Apr. 2018

\section{ABSTRACT}

Skin metastases from colorectal carcinoma are rare and signal advanced disease. Skin metastasis refers to growth of cancer cells in the skin originating from an internal cancer. In most cases, cutaneous metastasis develops after the initial diagnosis of the primary internal malignancy and late in the course of the disease. In very rare cases, skin metastasis may occur at the same time or before the primary cancer has been discovered and may be the prompt for further thorough investigation. The incidence of skin metastasis varies but is somewhere between $3-10 \%$ in patients with a primary malignant tumor. Most common ones are melanoma $(45 \%)$, breast cancer $(30 \%)$, nasal sinus cancers $(20 \%)$, cancer of the larynx and cancer of the oral cavity (12\%) cases. Here we present a case of 39-year-old man presented to us with multiple subcutaneous lesions over the chest and forehead. Fine needle aspiration cytology of skin nodules revealed metastatic adenocarcinoma features. Further imaging of the abdomen with computed tomography (CT) revealed circumferential narrowing of caecum and ascending colon. The patient was managed with palliative right hemicolectomy in view of constipation and palliative chemotherapy as systemic treatment. An extensive review of the English literature did not reveal extensive data on metastatic subcutaneous nodules leading to diagnosis of colon cancer.

\section{KEYWORDS:}

Subcutaneous nodules, Metastatic, Colon cancer

Please cite this paper as:

Madabhavi I, Kadakol N, Chavan C, Sarkar M. Multiple Subcutaneous Nodules Leading to Diagnosis of Colon Cancer. Middle East J Dig Dis 2018;10:188-191. doi: 10.15171/ mejdd.2018.109.

\section{INTRODUCTION}

The incidence of subcutaneous metastasis from a visceral malignancy, excluding malignant melanoma, lymphoma, and leukemia, is rare with an incidence of 5.3\%. ${ }^{1}$ Skin involvement as the presenting sign of a silent internal malignancy is an even rarer event occurring in approximately $0.8 \% .^{2}$ We report a rare case of a patient who presented to his dermatologist complaining of rapidly developing subcutaneous nodules which subsequently proved to be metastatic colon cancer, and we provide a review of the literature.

\section{CASE REPORT}

A 39-year-old man presented to a local dermatologist, with symptoms of multiple subcutaneous nodules over the anterior chest wall and 


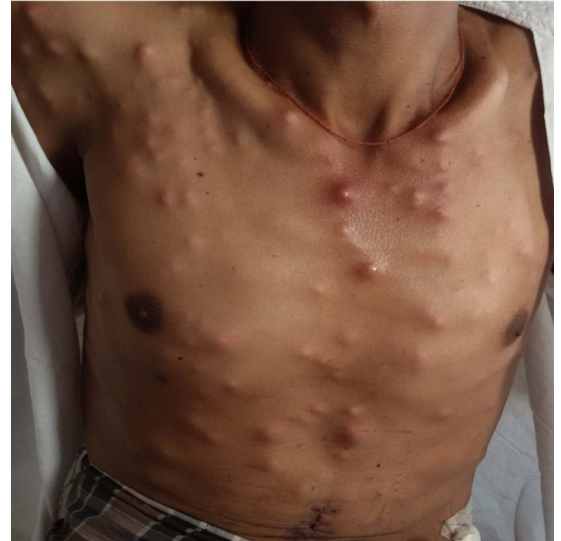

Fig.1: Multiple metastatic subcutaneous nodules over the anterior chest wall in a patient of colon cancer.

forehead for 3 months (figure 1). The patient also had history of significant weight loss and loss of appetite since last 3 months. He also had history of intermittent constipation lasting for 3-4 days since last 2 months. Fine needle aspiration cytology from skin nodules showed malignant cells with hyperchromatic nuclei, prominent nucleoli, and scanty cytoplasm, with some cells showing intracellular mucin collection suggestive of metastatic mucinous adenocarcinoma (figure 2). For expert opinion this patient came to our Cancer Care Centre.

On examination his vital signs were stable with performance status of 1 . His weight was $40 \mathrm{kgs}$, with emaciated, cachectic look and there was marked pallor. There was no associated lympadenopathy, icterus, or pedal edema. Multiple subcutaneous nodules were noted over the anterior abdominal wall, anterior chest wall, and forehead with size of 1-2 cm, firm too hard in consistency and non-tender (figure 1). There was no history of any bleeding per rectum, but the patient used to have altered bowel habits in the form of intermittent constipation.

Complete blood count showed hemoglobin of $8 \mathrm{gr} / \mathrm{dL}$, with normal leukocyte count of 5300 per cumm and platelet count of 350,000 per cumm. Carcinoembryonic antigen was $480 \mathrm{IU} / \mathrm{mL}$. Peripheral smear was suggestive of dimorphic anemia with MCV of 97 pg/mL. Contrast enhanced computed tomography (CT) of the abdomen showed

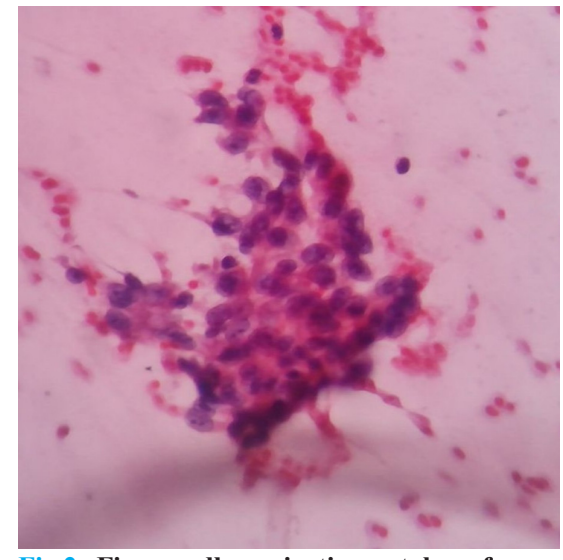

Fig.2: Fine needle aspiration cytology from skin nodules shows malignant cells with hyperchromatic nuclei with prominent nucleoli and scanty cytoplasm with some cells showing intracellular mucin collection.

asymmetrical circumferential thickening with luminal narrowing of caecum and ascending colon, extending to involve luminal narrowing of ascending colon near hepatic flexure with mild ascites without any evidence of liver metastasis (figure 3). Colonoscopy was done and biopsy samples were taken from the right sided ascending colonic mass. Histopathological image showed malignant cells with abundant mucinous cytoplasm and vesicular nuclei with hyperchromatism and prominent nucleoli. Tumor cells were arranged in glandular pattern with intraglandular mucin collection (figure 4) The patient was managed with blood transfusion, hematinics, and palliative right sided hemicolectomy in view of intermittent constipation. Postoperative chemotherapy with 5- fluorouracil, leucovorin, and oxiliplatin (FOLFOX) regimen was started and the patient is under regular treatment at our centre until date. Total 16 cycles of FOLFOX chemotherapy was completed without any major side effects except for the mild grade peripheral neuropathy. All the subcutaneous lesions have been regressed after 3 months of treatment and the current $\mathrm{CEA}$ (Carcinoembryonic antigen) is $56 \mathrm{IU} / \mathrm{mL}$.

\section{DISCUSSION}

Colorectal carcinoma most often metastasizes to liver and lung, while subcutaneous or skin metastases are rare, accounting for only $5 \%$ of such cases. ${ }^{3,4}$ Usually, for al- 


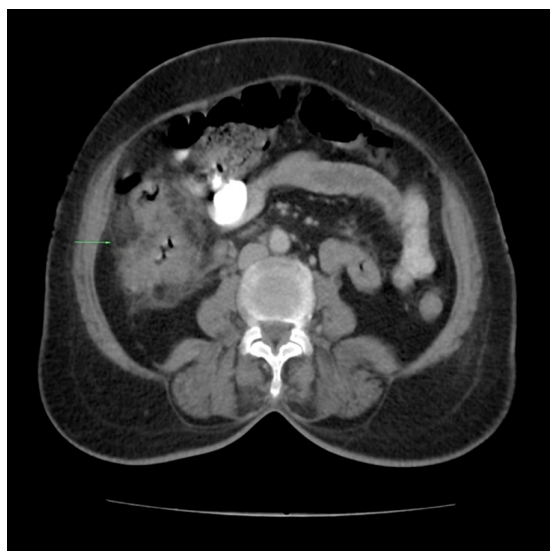

Fig.3: Contrast enhanced computed tomogram shows asymmetrical circumferential thickening with luminal narrowing of caecum and ascending colon.

most all cancers, skin involvement is synonymous with extensive disease and frequently implies a poor prognosis. In colorectal cancer, this event occurs mostly on the incision scars, but is also seen on the skin of the extremities, head and neck, and penis. ${ }^{5,6}$

Cutaneous metastasis may occur through lymphogenous spread, intravascular dissemination, direct extension of tumor, and surgical implantation. ${ }^{7}$ Wong and colleagues added the spread along embryonal remnants such as the urachus to the aforementioned mechanisms. ${ }^{8}$ With the current knowledge on molecular biology, there is not only the possibility of passive dissemination pathway, known as "tumor embolization", but also an easier way through the processes of angiogenesis and angiolymphatic growth factors that help to increase the total surface of blood vessels for tumor cell invasion. Following the blood vessel invasion, tumor cells that resist to the mechanic stress of circulation and to the immune system "colonize" the subcutaneous tissues until they acquire the necessary angiogenic properties and a favorable microenvironment to leave the state of "numbness".

It usually presents as violaceous to flesh-colored, firm, freely mobile, painless nodules, single or multiple (as inour case). It can sometimes mimic epidermal cysts, neurofibromas, lipomas, cicatricial morphea-like plaques, lymphoma, and alopecia. Survival after diagnosis of cutaneous metastasis ranges from 1 to 34 months. The average survival of patients after diagnosis of skin metastasis from colon cancer is, according to Lookingbill

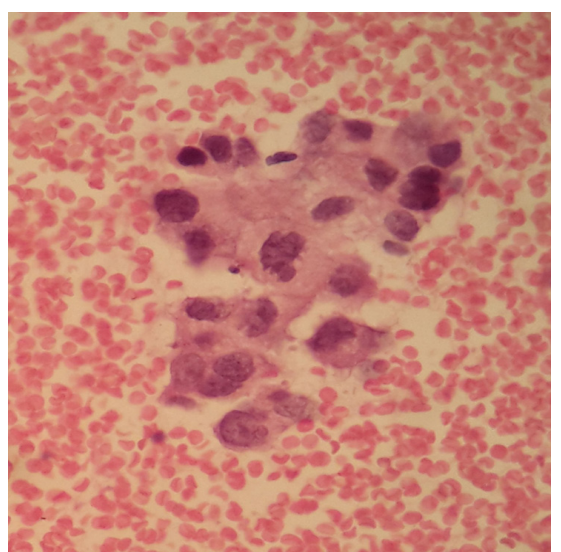

Fig.4: Histopathological H\&E image shows malignant cells with abundant mucinous cytoplasm and vesicular nuclei with hyperchromatism and prominent nucleoli. Tumor cells are arranged in glandular pattern with intraglandular mucin collection.

and co-workers, 18 months.

Wide local excision of the cutaneous metastatic lesion is the preferred treatment option in isolated lesions. In cases of extensive cutaneous metastases treatment is only palliative. No clear guidelines exist for an optimum chemotherapeutic regimen. The treatment of metastatic colorectal cancer has become increasingly complex and nuanced as treatments have evolved over the last decade. During that time, treatment has evolved from single agent 5-fluorouracil to combination chemotherapy and more recently the inclusion of monoclonal antibodies such as bevacizumab, aflibercept, ramucirumab, regorafenib, cetuximab, panitumab and recently immunotherapy with pembrolizumzb. As such, metastatic colorectal cancer is evolving into a chronic disease in which the median overall survival is in excess of 2 years and the 5-year survival is $10 \%$. Established chemotherapy include 5-fluorouracil, capecitabine, irinotecan, oxiliplatin, and cisplatin. Combinations of irinotecan to bolus 5-fluorouracil, oxaliplatin to infusional 5-fluorouracil, and leucovorin (FOLFOX) or infusional 5-fluorouracil/leucovorin with irinotecan (FOLFIRI) has increased survival to over 20 months. Treating patients sequentially with FOLFIRI followed by FOLFOX, or with FOLFOX followed by FOLFIRI, has increased the median survival times to 21.5 months and 20.6 months, respectively. ${ }^{10}$

\section{CONCLUSION}

In summary, cutaneous metastasis from a colorectal 
primary cancer is a rare event. When present, the metastasis usually signifies disseminated disease and poor prognosis. Further analysis of the biology of this mode of metastasis may shed light on mechanisms of metastasis to specific organs, and hopefully these findings will provide targets for novel targeted therapeutic intervention.

\section{ETHICAL APPROVAL}

There is nothing to be declared.

\section{CONFLICT OF INTEREST}

The authors declare no conflict of interest related to this work.

\section{ACKNOWLEDGEMENT}

All the authors express their heartfelt gratitude to the speedy recovery of the patient.

\section{REFERENCES}

1. Krathen RA, Orengo IF, Rosen T. Cutaneous metastasis: a meta-analysis of data. South Med J2003;96:1647. doi:10.1097/01.SMJ.0000053676.73249.E5.

2. Lookingbill DP, Spangler N, Sexton FM. Skin involvement as the presenting sign of internal carcinoma. A retrospective study of 7,316 cancer patients. J Am Acad Dermatol 1990;22:19-26. doi:10.1016/0190-9622(90)70002-Y.

3. Llaguna $\mathrm{OH}$, Desai $\mathrm{P}$, Fender AB, Zedek DC, Meyers MO, O'Neil BH, et al. Subcutaneous metastatic adenocarcinoma: an unusual presentation of colon cancer- case report and literature review. Case Rep Oncol 2010;3:386-90. doi:10.1159/000321948.

4. Horiuchi A, Nozawa K, Akahane T, Shimada R, Shibuya H, Aoyagi Y, et al. Skin metastasis from sigmoid colon cancer. Int Surg 2011;96:135-8. doi:10.9738/1391.1.

5. Stavrianos SD, McLean NR, Kelly CG, Fellows S. Cutaneous metastasis to the head and neck from colonic carcinoma. Eur J Surg Oncol 2000;26:518-9. doi:10.1053/ejso.1999.0935.

6. Camci C, Türk HM, Büyükberber S, Karakök M, Koruk M, Beyazity Y, et al. Colon carcinoma with synchronous subcutaneous and osseous metastasis: a case report. J Dermatol 2002;29:362-5. doi:10.1111/j.1346-8138.2002.tb00282.x.

7. Gu Y, Tang R, Gong DQ, Qian YL. Reconstruction of the abdominal wall by using a combination of the human acellular dermal matrix implant and an interpositional omentum flap after extensive tumor resection in patients with abdominal wall neo- plasm: a preliminary result. World $J$ Gastroenterol 2008;14:752-7.

8. Kauffman CL, Sina B. Metastatic inflammatory carcinoma of the rectum: tumor spread by three routes. Am J Dermatopathol 1997;19:528-32.

9. Andrade SMS, Pereira FL. [Synchronous colorectal cancer: a case report and literature review]. Rev bras Coloproct 2007;27:69-79. doi:10.1590/S010198802007000100010.

10. Tournigand C, André T, Achille E, Lledo G, Flesh M, Mery-Mignard D, et al. FOLFIRI followed by FOLFOX6 or the reverse sequence in advanced colorectal cancer: a randomized GERCOR study. J Clin Oncol 2004;22:229-37. doi:10.1200/JCO.2004.05.113. 